УДК 533.9, 539.1

\title{
VACUUM CREATION OF CHARGED PARTICLES: SOME INTEGRAL RELATIONS
}

\author{
A. V. Chertilin, A. G. Lavkin, R. S. Sitalieva, P. I. Smolyansky, \\ S. A. Smolyansky, A. V. Tarakanov \\ Saratov State University, Russia \\ E-mail: smol@sgu.ru
}

Dependence on the wave length and the periodical electric field strength of the densities of particle number and energy of the created from vacuum particle-antiparticle plasma of the fermi and bose types had investigated. Analytical and numerical calculations had fulfilled in the leading approximation for the case of rather small fields in comparison with the Schwinger critical field $E_{c}=m^{2} / e$.

Key words: vacuum creation, fermion, boson, kinetic equation, the number density, Schwinger effect.

\section{Вакуумное рождение заряженных частиц: некоторые интегральные соотношения}

\section{А. В. Чертилин, А. Г. Лавкин, Р. С. Ситалиева, П. И. Смолянский, С. А. Смолянский, А. В. Тараканов}

Изучена зависимость от длины волны и напряженности периодического электрического поля плотностей числа частиц и энергии, порожденной из вакуума плазмы, состоящей из частиц и античастиц фермиевского и бозевского типа. Аналитические и численные расчеты проведены в рамках лидирующего приближения в случае достаточно слабых полей по сравнению с критическим полем Швингера $E_{c}=m^{2} / e$.

Ключевые слова: вакуумное рождение, бозон, фермион, кинетическое уравнение, плотность числа частиц, эффект Швингера.

\section{Introduction and Statement of the Problem}

The kinetic description of the vacuum particle creation under action of the quasi-classical field of different physical nature had obtained wide spread occurrence in the physics of heavy ion collisions, in the strong laser fields and cosmology (see the review works [1-4]). In the case of the electromagnetic field, as a rule, the linear polarized time dependent space homogeneous electric field is considered, $A^{\mu}(t)=$ $=\left(0,0,0, A^{3}(t)=A(t)\right)$. For such fields the kinetic equation has the simplest form and admits the solution in the low density approximation,

$$
f(\mathbf{p}, t)=\frac{1}{4}\left|\int_{t_{0}}^{t} d t^{\prime} \lambda\left(\mathbf{p}, t^{\prime}\right) e^{2 i \theta\left(t^{\prime}, t_{0}\right)}\right|^{2} .
$$

It is assumed, that the distribution function $f(\mathbf{p}, t)$ is rather small, $f(\mathbf{p}, t)<<1$. The amplitude $\lambda$ $(\mathbf{p}, t)$ has different structure for the fermions $(f)$ and the scalar bosons $(b)$,

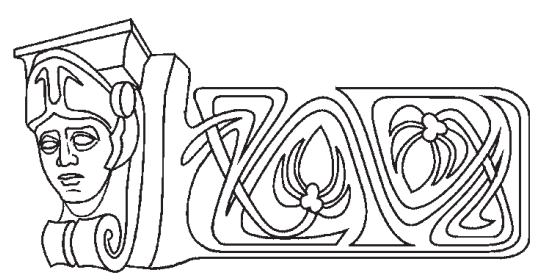

$$
\lambda_{i}(\mathbf{p}, t)=\frac{e E(t) w_{i}(\mathbf{p})}{\omega^{2}(\mathbf{p}, t)},
$$

where $E(t)=-A(t)$ is the field strength and $(i=f, b)$,

$$
w_{i}(\mathbf{p})=\left\{\begin{array}{l}
\varepsilon_{\perp}(\mathbf{p})=w_{f}, \\
P=w_{b},
\end{array}\right.
$$

$\varepsilon_{\perp}(\mathbf{p}, t)=\left(m^{2}+p_{\perp}^{2}\right)^{1 / 2}$ is the transversal energy $\left(p_{\perp}=\left(p_{1}^{2}+p_{2}^{2}\right)^{1 / 2}\right), P=p_{\|}-e A(t)$ is the kinematic momentum and $\omega(\mathbf{p}, t)=\left[\varepsilon_{\perp}^{2}(p)+P^{2}\right]^{1 / 2}$ is the quasi-energy.

For the periodical electric («laser») field

$$
A(t)=\left(E_{0} / v\right) \cos v t, \quad E(t)=E_{0} \sin v t
$$

( $v$ is the angular frequency) on the basis of Eq. the following solution was obtained in the leading approximation relatively of the electric field :

$$
\begin{gathered}
f(\mathbf{p}, t)=f_{0}(\mathbf{p})+\frac{1}{2} f_{2}(\mathbf{p})[1-\cos 2 v t] \\
f_{0 i}(\mathbf{p})=\pi \alpha E_{0}^{2} \frac{v^{2} w_{i}^{2}(\mathbf{p})}{\omega_{0}^{4}(\mathbf{p})\left[4 \omega_{0}^{2}(\mathbf{p})-v^{2}\right]^{2}} \\
f_{2 i}(\mathbf{p})=\pi \alpha E_{0}^{2} \frac{w_{i}^{2}(\mathbf{p})}{\omega_{0}^{4}(\mathbf{p})\left|4 \omega_{0}^{2}(\mathbf{p})-v^{2}\right|}
\end{gathered}
$$

Here we used the notations: $\omega_{0}(\mathbf{p})=\left.\omega(\mathbf{p}, t)\right|_{E=0}$ and $\alpha=e^{2} / 4 \pi$. The resonance condition $\mathrm{V}=$ $=2 \omega_{0}(\mathbf{p})$ shows on the one photon character excitation of the particle-antiparticle plasma. For the bosons according to Eq.(3) $w_{b 0}=\mathrm{P}_{\|}$.

In the present work, on the basis of Eqs. (5)-(7) some physical observables will be obtained, both for the fermionic and the bosonic fields. The question is about the particle number density of the quasiparticles

$$
n(t)=g \int \frac{d^{3} p}{(2 \pi)^{3}} f(\mathbf{p}, t)
$$

( $g$ is the degeneration factor: $g_{f}=4$ and $g_{b}=2$ for the particles with the lowest spins), the energy density

$$
\varepsilon(t)=g \int \frac{d^{3} p}{(2 \pi)^{3}} \omega(\mathbf{p}, t) f(\mathbf{p}, t) .
$$




\section{The Integral Observables}

We will consider the fermion and boson cases separately.

\section{The Fermion Case}

Firstly let us calculate the number density (8) of the electron-positron plasma (EPP). Performing the transition to the cylindrical coordinate system,

$$
\int d^{3} p=2 \pi \int_{-\infty}^{\infty} d p_{\mathrm{P}} \int_{0}^{\infty} p_{\perp} d p_{\perp},
$$

and substituting the functions (6) and (7) into Eq.(8), we obtain

$$
n(t)=n_{0}+\frac{1}{2} n_{2}[1-\cos 2 v t],
$$

where $(b=v / 2 m)$,

$$
\begin{aligned}
& n_{0}^{f}=\alpha E_{0}^{2} \frac{-4 \sqrt{\left|1-b^{2}\right|}+4-2 b^{4}-b^{2}\left(3 \sqrt{\left|1-b^{2}\right|}-1\right)}{24 b^{4} m \sqrt{\left|1-b^{2}\right|}}, \\
& n_{0}^{f}=\alpha E_{0}^{2} \frac{-4 \sqrt{\left|1-b^{2}\right|}+4-2 b^{4}-b^{2}\left(3 \sqrt{\left|1-b^{2}\right|}-1\right)}{24 b^{4} m \sqrt{\left|1-b^{2}\right|}},
\end{aligned}
$$

In the leading approximation relatively $b<<1$ (this condition is a consequence of the low density approximation $f<<1$ ) we obtain from here

$$
n_{0}^{f}=\frac{5 \alpha}{3 \cdot 2^{8} m} E_{0}^{2} \frac{v^{2}}{m^{2}}, \quad n_{2}^{f}=\frac{3 \alpha}{32 m} E_{0}^{2} .
$$

The resonance denominators in Eqs. (11), (12) show also on the one photon mechanism of EPP excitation.

Dependence $n_{0}^{f}(\lambda)$ and $n_{2}^{f}(\lambda)$ are depicted on Fig. 1 . In the «dangerous» point $b=1$ ( (it corresponds to the energy condition of the one EPP creation, $v=2 m)$ the density $n_{2}^{f}(\lambda)$ remains limited while $n_{0}^{f}(\lambda)$ is displayed the break stipulated by absence of the damping mechanism in the considered approximation.

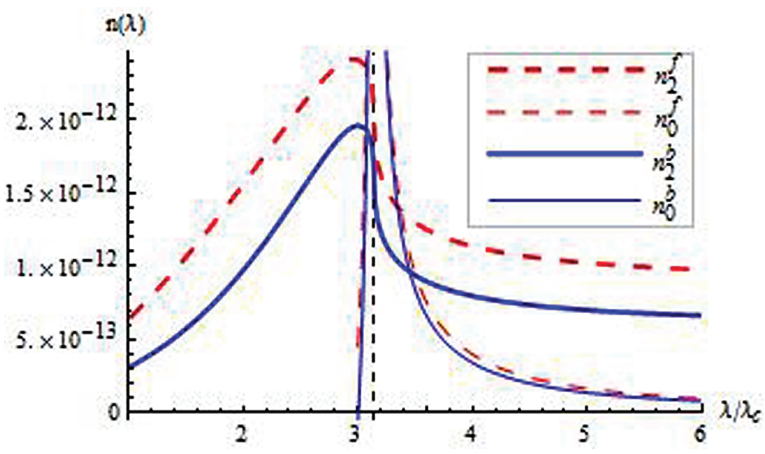

Fig.1. The number densities as functions $\lambda$ of the EPP and boson plasma for $\mathrm{E} 0=3 \cdot 10^{-5} E_{c r}$

The frequency dependence of the energy density has the form similar to Eq.(10),

$$
\varepsilon(t)=\varepsilon_{0}+\frac{1}{2} \varepsilon_{2}[1-\cos 2 v t],
$$

where now

$$
\varepsilon_{0}^{f}=\frac{\alpha E_{0}^{2}}{\pi} \cdot \frac{3 \arctan \left(b / \sqrt{\left|1-b^{2}\right|}\right)+\left(3 b+2 b^{3}\right) \sqrt{\left|1-b^{2}\right|}}{12 b^{3} \sqrt{\left|1-b^{2}\right|}} .
$$

The integral $\varepsilon_{2}^{f}$ has the logarithmic divergence. The regularization procedure in such kind divergent integral is not uniquely defined (the cut off over upper momentum, a few variants of the counter-term method) and leads to some different results. We will use the method based on subtraction of the leading terms at $p \rightarrow \infty$. The $p$-dependent part of the numerator in the integrand function Eq.(9) is equal to $\varepsilon_{\perp}^{2}(\mathbf{p})$. The relevant reduction of the divergence degree can be reached by means of elimination here $p^{2}$-dependence. Thus, the regularized expression for the energy density of the oscillation part EPP energy will be equal then

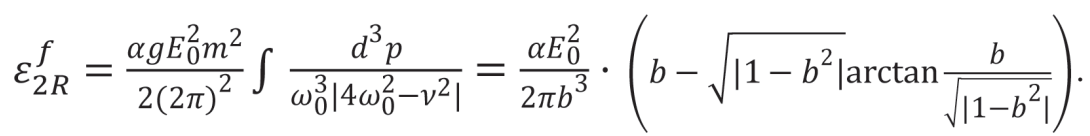

\section{The Boson Case}

Let us calculate now the boson particle number density similar to the fermion case

$$
n_{0}^{b}=\alpha E_{0}^{2} \frac{-8 \sqrt{\left|1-b^{2}\right|}+8-b^{2}\left(4+b^{2}\right)}{24 b^{4} m \sqrt{\left|1-b^{2}\right|}},
$$

$$
n_{0}^{b}=\alpha E_{0}^{2} \frac{-8 \sqrt{\left|1-b^{2}\right|}+8-b^{2}\left(4+b^{2}\right)}{24 b^{4} m \sqrt{\left|1-b^{2}\right|}}
$$

Fig. 1 shows the particle number density boson and fermion for comparison. 
The corresponding energy density has the same time dependence (15), where now

$$
\varepsilon_{0}^{b}=\frac{\alpha E_{0}^{2} b^{2}}{4 \pi}\left(-6 \sqrt{\left|1-b^{2}\right|} \arctan \frac{b}{\sqrt{\left|1-b^{2}\right|}}-2 b\left(-3+b^{2}\right)\right) .
$$

The time dependent part of the energy density is diverged also (the logarithmic divergence) as in the fermionic case. However, the counter-term procedure leads (in the leading approximation) to nulling of the corresponding integral, i.e.

$$
\varepsilon_{2 R}^{b}=0 \text {. }
$$

Some actual time dependence appears here in the next order $\left(\sim \alpha^{4}\right)$ of the perturbation theory, that corresponds to the 4 -th harmonics in $\varepsilon_{R}^{b}(t)$ spectrum.

\section{Summary}

We had investigated some features in behaviour of the particle number and energy densities of the fermion and boson particle-antiparticle gases created from vacuum under action of a strong periodical electric field. We had limited by the multiphoton domain $\gamma>>1$ only ( $\gamma$ is the adiabaticity parameter). Figs. 1 and 2 demonstrate the well known fermion domination in the theory of vacuum particle creation [6] .

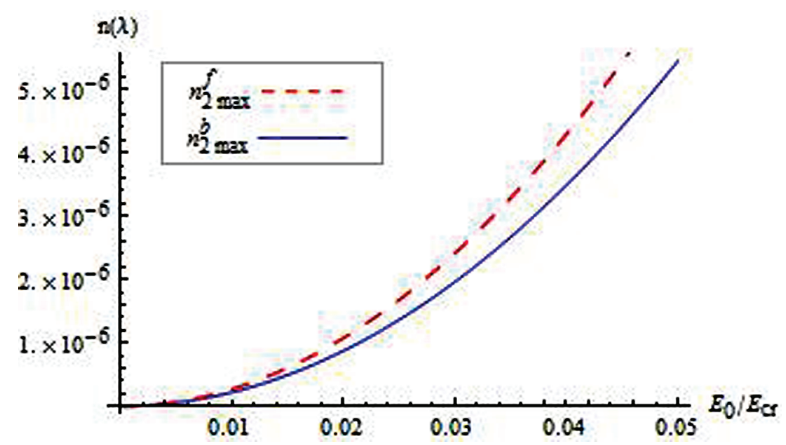

Fig.2. The dependence $n_{\max }\left(E_{0}\right)$ for the bosons and fermions

Position of the resonance points of the curves $n_{0,2}^{i}(\lambda)$ (Fig.1) is rather unexpected: in shifts considerably sideways long waves relatively of the point $\lambda=\pi \lambda_{c}$ which corresponds to the one photon creation mechanism.
A consequence of the conducted analysis is understanding of the fact that the distribution functions (5)-(7) (obtained as a result of the perturbation theory) have not «good» asymptotics at $p \rightarrow \infty$ that leads to the divergence of the energy density and the all highest moment.

The entropy calculated on the basis of Eqs. (6) and (7) has a complicated oscillator character.

The authors are grateful Prof. A. V. Prozorkevich for useful remarks.

\section{References}

1. Vinnik D. V., Miserny V. A., Prozorkevich A. V., Smolyansky $S$. A., Toneev $V$. D. Kinetic description of vacuum particle production in collisions of ultrarelativistic nuclei // Physics of Atomic Nuclei. 2001. Vol. 64. P. 775-786.

2. Blaschke D. B., Prozorkevich A. V., Röpke G., Roberts C. D., Schmidt S. M., Shkirmanov D. S., Smolyansky S. A. Dynamical Schwinger effect and high-intensity lasers. Realising of nonperturbative QCD // Eur. Phys. J. D. 2009. Vol. 55. P. 341-358.

3. Filatov A. V., Prozorkevich A. V., Smolyansky S. A., Toneev $V$. D. Inertial Mechanism : Dynamical Mass as a Source of Particle Creation // Physics of Elementary Particles and Atomic Nuclei. 2008. Vol. 39. P. 1721-1770.

4. Smolyansky S. A., Blaschke D. B., Tarakanov A. V., Schmidt S. M. Vacuum particle creation: statistical description // Applied Nonlinear Dynamics. 2009. Vol. 17. P. 17-29 (in russian).

5. Smolyansky S. A., Blaschke D. B., Fedotov A. M., Lavkin A. G., Prozorkevich A. V. Short Distance lectromagnetic Response of the Physical Vacuum. 2011. Workshop PWL 2011, Dresden, Sept. 5-9. URL: www.hzdr.de. (дата обращения: 01.06.2012).

6. Grib A. A., Mamaev S. G. and Mostepanenko V. M. Vacuum Quantum Effects in Strong External Fields. Friedmann Laboratory Publishing. St. Petersburg, 1994. 